\title{
Inducible Nitric Oxide Expression Correlates with the Level of Inflammation in Periapical Cysts
}

Mariza Akemi Matsumotoa, Daniel Araki Ribeirob

\begin{abstract}
Objectives: In an attempt to elucidate if inducible nitric oxide expression (iNOS) is correlated with the level of inflammation in periapical cysts with accuracy, the goal of this study was to evaluate the expression of iNOS in these ones.

Methods: 30 cases were included in this study being iNOS evaluated by means of immunohistochemistry. Statistical analysis was performed by Kruskal-Wallis non-parametric test followed by the post-hoc Dunn's test.

Results: iNOS stain was detected throughout the epithelium, subepithelial fibroblasts and macrophages in all cases, indistinctly. Nevertheless, iNOS immunostaining in periapical cysts was different according to the levels of inflammation, being the strongest effect associated with intense inflammatory infiltrate.

Conclusions: Taken together, our results indicate that immunoreactivity of iNOS was expressed in several cellular types present in periapical cyst, being positively correlated with the level of inflammation. Therefore, iNOS expression plays an important role in the pathogenesis of periapical cysts. (Eur J Dent 2007;1:212-215)
\end{abstract}

Key words: Periapical cyst; iNOS; Inflammation; Periapical cyst; Immunoreactivity.

\section{INTRODUCTION}

Periapical cysts are the most common cystic lesions of the jaws. ${ }^{1}$ They represent a periapical inflammatory disease caused by infected and necrotic dental pulp as a result of dental caries. ${ }^{2}$ In these circumstances, Malassez epithelial rests proliferate and finally form the lining epithelium of periapical cysts. $^{3}$

a Department of Oral and Maxillofacial Surgery, University of Sacred Heart, USC, Bauru, SP, Brazil

${ }^{b}$ Department of Biosciences, Federal University of São Paulo, UNIFESP, Santos, SP, Brazil

- Corresponding Author: Daniel Araki Ribeiro, DDS, PhD Departamento de Biociências Universidade Federal de São Paulo - UNIFESP Av. Ana Costa, 95 Vila Mathias Santos - SP Brazil Zip code: 11060-001

Phone: 551332222048 Fax: 551332222048 e-mail: daribeirođunifesp.br
Several inflammatory mediators widely known such as protaglandins and leukotrienes exert diverse physiological actions in different tissues and are closely involved into the pathogenesis of periapical disorders. ${ }^{4}$ Nitric oxide is an important signaling messenger that has been shown to play important role in many physiological and pathological conditions, such as inflammation. ${ }^{5}$ Endogenous nitric oxide is generated from Larginine by three major types of nitric oxide synthesis, endothelial, neural and inducible nitric oxide (iNOS). ${ }^{6}$ Particularly, iNOS produces much larger amounts of nitric oxide and has been detected in many inflammatory diseases, including those affecting oral tissues. ${ }^{7}$ However, data about the real significance of iNOS expression 
in periapical cysts following inflammatory process are fairly limited in literature. 8,9 As a result of limited evidence, the present study was undertaken to investigate if inducible nitric oxide expression (iNOS) is correlated with the level of inflammation in periapical cysts. Certainly, further knowledge on iNOS may be important for understanding the molecular mechanisms of periapical cysts pathogenesis.

\section{MATERIALS AND METHODS}

Cases

All experimental protocols used in this study were approved by the Ethical Committee for Human Research, University of Sacret Heart (USC), Bauru, SP, Brazil. All specimens were retrieved from the Dental Clinics, School of Dentistry, University of Sacret Heart (USC), Bauru, SP, Brazil. The tissues had been fixed in $10 \%$ buffered formalin. 30 cases were selected under histopathological analysis (21 males and 9 females) ranging in age from 22 to 58 years. No systemic disease was observed in any patients.

All patients underwent cyst enucleation at the Clinics of Oral Surgery, University of Sacret Heart (USC), Bauru, SP, Brazil. Histological diagnoses were based on the following criteria: fully developed cavities lined by stratified squamous epithelium with variable thickness and a fibrous capsule. ${ }^{1}$ This was made by two of the authors in conjunction (MAM and DAR).

\section{Immunohistochemistry}

Serial sections of $4 \mu \mathrm{m}$ were deparaffinized in xylene and rehydrated in graded ethanol, then pretreated in a microwave with $10 \mathrm{mM}$ citric acid buffer ( $\mathrm{pH}=6)$ for 3 cycles of 5 min each at $850 \mathrm{~W}$ for antigen retrieval. They were pre-incubated with $0.3 \%$ hydrogen peroxide in phosphate buffered saline (PBS) for 5 min for inactivation of endogenous peroxidase, and then blocked with $5 \%$ normal goat serum in PBS for $10 \mathrm{~min}$. The specimens were then incubated with anti-iNOS monoclonal antibody (N-19, Santa Cruz Biothechnology, USA), at a concentration of $1: 400$. All incubations were carried out overnight at $4^{\circ} \mathrm{C}$ within the refrigerator. This was followed by two washes in PBS for 10 $\min$. The sections were then incubated with biotinconjugated secondary antibody anti-mouse IgG (Vector Laboratories, Burlingame, CA, USA) at a concentration of $1: 200$ in PBS for 1 h. After that, the sections were washed twice with PBS followed by the application of preformed avidin biotin complex conjugated to peroxidase (Vector Laboratories, Burlingame, CA, USAl for $45 \mathrm{~min}$. The bound complexes were visualized by the application of a $0.05 \%$ solution of $3-3$-diaminobenzidine solution, and counterstained with methyl green. For control studies of the antibodies, the serial sections were treated with mouse IgG in place of the primary antibody. Additionally, internal positive controls were performed with each staining batch.

\section{Data analysis}

Hematoxylin eosin (H.E.) sections were examined blindly by two of the authors in conjunction (DAR and MAM) in 3-5 randomly selected microscopic fields at $x 400$ magnification in order to quantify the grade of inflammation present in all periapical cysts studied. The percentages of inflammatory cells were categorized, as follows: intense - more than $50 \%$ of cells present; moderate $-25 \%$ to $49 \%$ positive cells and mild - 0 to $24 \%$ cells within the fields. Occasional disagreements regarding the classification were discussed and a consensus reached.

Sections stained with immunohistochemistry were analyzed for the percentages of immunopositive cells under the optical microscope. A total of 500-1000 cells were evaluated blindly in 3-5 fields at $x 400$ magnification. These values were used as labeling indices.

\section{Statistical methods}

Statistical analysis was performed by KruskalWallis non-parametric test followed by Dunn's test if a significant effect was detected using SPSS software pack (version 1.0). A $P$ value $<.05$ was considered for statistical significance.

\section{RESULTS}

Under light microscopic examination, H.E. sections showed the typical morphology of a periapical cyst. The cystic lumen was partially or entirely lined by non-keratinized stratified squamous epithelium. The underlying fibrous connective tissue wall was inflamed with variable degree of inflammatory cell infiltration, being four cases $(13 \%)$ presenting mild inflammation and nine $(30 \%)$ and $17(57 \%)$ with moderate and 
severe inflammation, respectively. Many small blood vessels were scattered within the cystic connective wall, specially concentrated in the regions presenting inflammatory infiltrate.

iNOS stain was detected throughout the epithelium and subepithelial fibroblasts, and inflammatory cells in all cases indistinctly. Nevertheless, iNOS immunostaining in periapical cysts was different according to the levels of inflammation observed. For example, a statistically significant increase $(P<.05)$ of iNOS expression was observed in periapical cysts with higher levels of inflammation when compared to the tissues with low levels of inflammatory infiltrate. Such data are presented in Figure 1. No immunoreactivity was detected in the negative control.

\section{DISCUSSION}

The aim of this study was to evaluate if inducible nitric oxide expression (iNOS) is correlated with the level of inflammation in periapical cysts by means of immunohistochemistry. Periapical cysts are believed to result from continuous antigenic stimulation from inflamed or necrotic root canals. ${ }^{1}$ All cases selected in this study exhibited typical features of periapical cysts such as complete epithelial lining, surrounding collagen fibers and granulomatous tissues infiltrated with a number of inflammatory cells at different levels.

Accumulating evidencesuggeststhat nitricoxide has a variety of biological activities, including the inhibition of neutrophil adhesion to endothelium; ${ }^{10}$ the dysfunction of lysosomal enzyme release; ${ }^{11}$ and a decrease of neutrophil chemotaxis. ${ }^{12}$ Furthermore, it has been demonstrated that nitric oxide induces apoptosis, intracellular iron loss and also inhibits mitochondrial function and DNA

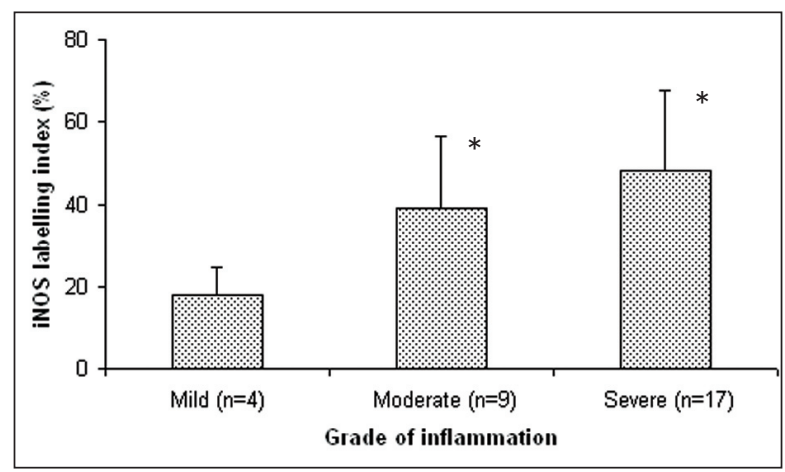

Figure 1. iNOS labeling index in reduced epithelium and stratified squamous epithelium of dental follicles. Values were expressed as means \pm S.D. *: P<.05 when compared to mild inflammation. synthesis. ${ }^{13-15}$ Particularly, the inducible isoform of nitric oxide synthase (iNOS) is a calciumindependent cytosolic enzyme induced mainly by certain cytokines and bacterial lipopolysaccharides at sites of inflammation. ${ }^{16}$ Recent studies suggest that activation of iNOS is closely related to the pathophysiologic characteristics of inflammatory diseases. ${ }^{8}$ Despite the rapid growth in the field of nitric oxide research, the role of this free radical in periapical cysts has not still been elucidated, mainly during inflammatory process. In this study, histological evidence is presented which supports the finding that iNOS expression is upregulated in periapical cysts. In cystic tissues, epithelial cells and fibroblasts were significantly stained with iNOS antibodies. Inflammatory cells, including polymorphonuclear (PMN) and mononuclear cells also showed iNOS reactivity. These results are fully in line with previous reports. 8,9

It is well established that nitric oxide plays an important role in host defense and homeostasis when generated at a low level for a brief period of time, whereas the prolonged induction of nitric oxide levels may contribute to a variety of pathological phenomena associated with inflammatory processes. ${ }^{17,18}$ In this study, we found high nitric oxide levels in periapical cysts with high degree of inflammation. On the basis of these data, we assumed that iNOS is able to modulate inflammation as far as to induce substantial tissue damage circumjacent to periodontal tissues leading to cystic growth.

\section{CONCLUSIONS}

Our results indicate that immunoreactivity of iNOS was expressed in several cellular types present in periapical cyst. iNOS expression was positively correlated with the level of inflammation. Therefore, iNOS expression plays an important role in the pathogenesis of periapical cysts.

\section{REFERENCES}

1. Shear M. Cysts of the jaws: recent advances. J Oral Pathol 1985; 14:43-49.

2. Meghji S, Qureshi W, Henderson B, Harris M. The role of endotoxin and cytokines in the pathogenesis of odontogenic cysts. Arch Oral Biol 1996;41:523-531.

3. Shafer WG, Hine MK, Levy BM. Diseases of the pulp and periapical tissues. A textbook of oral pathology. Philadelphia, W.B. Saunders Company, 1983;479-510. 
4. Stashenko P, Teles R, D'Souza R. Periapical inflammatory responses and their modulation. Crit Rev Oral Biol Med 1988;9:498-521.

5. Fukumura D, Kashiwagi S, Jain RK. The role of nitric oxide in tumour progression. Nature Reviews Cancer 2006;6:521534.

6. Knowles RG, Moncada S. Nitric oxide syntheses in mammalian cells. Biochem J 1994;298:249-258.

7. Hsu YY, Jou YT, Wong R, Karabucak B, Simchon S, Kim S. Effect of nitric oxide synthase inhibitor (L-NAME) on substance P-induced vasodilatation in the dental pulp. Int Endod J 2003;36:840-847.

8. Takeichi O, Hayashi M, Tsurumachi T, Tomita H, Ogihara B, Ogiso $B$, Saito $T$. Inducible nitric oxide synthase activity by interferon- $\tau$-producing cells in human radicular cysts. Int Endod J 1999;32:124-130.

9. Suzuki T, Kumamoto H, Doya K, Motegi K. Expression of inducible oxide synthase and heat shock proteins in periapical inflammatory lesions. J Oral Pathol Med 2002;31:488-493.

10. Kubes $P$, Suzuki M, Granger DN. Nitric oxide an endogenous modulator of leukocyte adhesion. Proc Natl Acad Sci 1991;88:4651-4655.

11. SchroderH, NeyP, Woditsh I,SchororK. Cyclic GMPmediates SIN-1-induced inhibition of human polymorphonuclear leukocytes. Eur J Pharmacol 1991;182:211-218.

12. Kaplan SS, Biliar T, Curran RD, Adiziarski VE, Simmon RL, Besford RE. Inhibition of chemotaxis with NG-monomethylL-arginine: a role for cyclic GMB. Blood 1989;74:18851887.

13. Alvina JE, Cui S, Mateo RB, Reicher JS. Nitric oxide mediated apoptosis in murine peritoneal macrophages. $J$ Immunol 1993;150:5080-5085.

14. Stuehr DJ, Nathan CF. Nitric oxide. A macrophage product responsible for cytostasis and respiratory inhibition in tumor target cells. J Exp Med 1989;169:1543-1555.

15. Lepoivre M, Coves J, Fontecave M. Inhibition of ribonucleotide reductase by nitric oxide. In: Moncada S, Marletta MA, Hibbs Jr JB, Higgs EA. Eds. Biology of Nitric Oxide, part 2. 1992; London UK, Portland Press.

16. Breft DS, Hwang PM, Glatt CE, Lowenstein C, Reed RR, Snyder SH. Cloned and expressed nitric oxide synthase structurally resembles cytochrome $\mathrm{p}-450$ reductase. Nature 1991;351:714-718.

17. Beevi SS, Rasheed AM, Geetha A. Evaluation of oxidative stress and nitric oxide levels in patients with oral cavity cancer. Jpn J Clin Oncol 2002;24:379-385.

18. Nathan C, Xie QW. Nitric oxide synthases: roles, tolls, and controls. Cell 1994;78:915-918. 